ESCRTS AND ASSOCIATED PROTEINS IN LYSOSOMAL FUSION WITH ENDOSOMES AND AUTOPHAGOSOMES

\begin{tabular}{|r|l|}
\hline Journal: & Biochemistry and Cell Biology \\
\hline Manuscript ID & bcb-2016-0099.R2 \\
\hline Manuscript Type: & Mini Review \\
\hline Date Submitted by the Author: & 16 -Jul-2016 \\
\hline Complete List of Authors: & $\begin{array}{l}\text { Majumder, Priyanka; Saha Institute of Nuclear Physics, Biophysics and } \\
\text { Structural Genomics } \\
\text { Chakrabarti, Oishee; Saha Institute of Nuclear Physics, Biophysics and } \\
\text { Structural Genomics }\end{array}$ \\
\hline Keyword: & $\begin{array}{l}\text { ESCRT, ESCRT regulatory proteins, Autophagosome, Endosome, Lysosomal } \\
\text { fusion }\end{array}$ \\
\hline &
\end{tabular}




\title{
ESCRTS AND ASSOCIATED PROTEINS IN LYSOSOMAL FUSION WITH ENDOSOMES AND AUTOPHAGOSOMES
}

\author{
Priyanka Majumder, Oishee Chakrabarti
}

Biophysics and Structural Genomics Division, Saha Institute of Nuclear Physics, Kolkata, India.

Correspondence: O Chakrabarti, Biophysics and Structural Genomics Division, Saha Institute of Nuclear Physics, Sector-1, Block-AF, Bidhannagar, Kolkata, West Bengal 700064, India. Tel: +91332337 5345; Fax: +913323374637

E-mail: oishee.chakrabarti@saha.ac.in

Key words: ESCRT, ESCRT regulatory proteins, Lysosomal fusion, Autophagosome, Endosome. 


\begin{abstract}
:
Endo-lysosomal and autophagosomal degradation pathways are highly connected at various levels, sharing multiple molecular effectors that modulate them individually or simultaneously. These two lysosomal degradative pathways are primarily involved in the disposal of cargo internalized from the cell surface or long lived proteins or aggregates and aged organelles present in the cytosol. Both these pathways involve a number of carefully regulated vesicular fusion events which are dependent on ESCRT proteins. The ESCRT proteins especially ESCRT-I and III participate in the regulation of fusion events between autophagosome/amphisome and lysosome. Along with these, a number of functionally diverse ESCRT associated and regulatory proteins such as, endosomal PtdIns (3) P 5-kinase Fab1, ALIX, Mahogunin RING Finger1, Atrogin1, Syntaxin17, ATG12-ATG3 complex and Protein kinase CK2 $\alpha$ are involved in fusion events in either or both the lysosomal degradative pathways.
\end{abstract}




\section{Introduction:}

Lysosome is the terminal degradative organelle in a cell. It degrades macromolecules from the endocytic and autophagic pathways (Huotari and Helenius 2011; Fader and Colombo 2009; Hyttinen et al. 2013). The targeting of vesicles to lysosomes involves a number of processes that are orchestrated in a regulated manner. A plethora of proteins are involved in fusion events of (i) endosomes with lysosomes and (ii) autophagosomes with lysosomes (Luzio et al. 2010; Ganley. 2013, Moreau et al. 2013 , Metcalf and Isaacs 2010; Hyttinen et al. 2013; Lu et al. 2013).

In the endocytic pathway, ubiquitinated membrane bound proteins and growth factors are captured and internalized in early endosomes which subsequently mature into late endosomes and multivesicular bodies (MVBs). They then fuse with lysosomes for degradation of cargo (Huotari and Helenius 2011). While, in the autophagic pathway, long lived proteins, aggregates and aged organelles are sequestered in double membranous autophagosomes which then fuse with late endosomes to form amphisomes for subsequent fusion with lysosomes (Fader and Colombo 2009; García-Arencibia et al. 2010). Autophagosomes may also directly fuse with lysosomes. All these fusion processes are highly regulated, finely orchestrated by a cascade of molecular players from different protein families.

There is a rich literature on the role of ESCRT (endosomal sorting component required for transport) proteins in the formation and maturation of endosomes (Raiborg and Stenmark 2009; Huotari and Helenius 2011; Gruenberg and Stenmark 2004; Bache et al. 2003; Babst et al. 2002). The role played by these molecules is also progressively being understood in the autophagy pathway (Rusten and Simonsen 2008; Fader and Colombo 2009; Rusten and Stenmark 2009; Metcalf and Isaacs 2010; Manil-Segalén et al. 2012). The ESCRT pathway proteins have been also implicated in multiple other cellular mechanisms. During cytokinesis, once the midbody has thinned, these proteins mediate the final membrane scission event that separates the two daughter cells (Agromayor and Martin-Serrano, 2013; Henne et al. 2013; Caballe et al. 2015). There is also quite an extensive literature to demonstrate that the ESCRT components actively participate in exosome formation and viral budding (Carlton and Martin-Serrano 2007; Votteler and Sundquist 2013; Bartusch and Prange 2016; Hurley 2015). However, our understanding is still far from clear about the effect of ESCRT regulatory proteins that modulate fusion of various vesicles with the lysosomes, with emphasis on autophagosomal-lysosomal fusion events (Lu et al. 2013; Rusten et al. 2007; Majumder and Chakrabarti 2015). An understanding of the ESCRT 
regulatory proteins in vesicular fusion events cannot be undermined because they play an essential role in modulating the function of the ESCRT proteins in guiding these events. The ESCRTs and their accessory proteins participate in a number of fusion processes (Box 1). To analyze the role of the ESCRT regulatory proteins in lysosomal fusion events, it is first imperative to understand how these events are affected by the ESCRT proteins. This review is hence primarily divided into two sections - first we discuss the general structure of lysosomal degradative pathway with role of the ESCRTs in the endocytic and autophagic processes. The second section enumerates the different ESCRT regulatory proteins and discusses their role in fusion of endosomes/amphisosomes/autophagosomes with lysosomes. These associated proteins alter ESCRTs via multiple modes, some of which may be at the genetic level (Lu et al. 2013) or via post-translational modifications (Majumder and Chakrabarti 2015; Kim et al. 2007).

\section{Pathways feeding cargo to the lysosomes}

Macromolecules intended for degradation at the lysosomes, reach their final destination through multiple pathways, though the role of ESCRTs is primarily implicated in the endo-lysosomal and autophago-lysosomal pathways. Along with this, recent literature suggests that these proteins also regulate a junction point - the amphisosmes - where both these pathways may choose to converge before fusing with the lysosomes.

\subsection{Endocytic degradative pathway and ESCRTs}

Endocytic pathway functions to maintain the normal cellular housekeeping by importing macromolecules into the cells from outside, followed by their transport, sorting and degradation. The pathway acts complementary to the autophagic pathway. Endocytosed materials, such as fluid solutes, macromolecules, particles, and membrane receptors are internalized into vesicles coated with any of these following proteins such as clathrin, caveolin, CLIC/GEEC (clathrinindependent carriers/GPI-enriched early endosomal compartments) and ADP-ribosylation factor 6(ARF6) (Huotari and Helenius 2011; Mayor and Pagano 2007). The vesicles then either fuse with each other to form early endosomes or can fuse with preexisting small GTPases RAB5 positive early endosome (Huotari and Helenius 2011). Early endosomes act as sorting vesicles from where cargoes are transferred back to cells membrane, or targeted to trans golgi network (TGN) or sorted to late endosomes for final degradation in lysosome (Huotari and Helenius 
2011; Spang 2009). Proteins, such as receptors for iron-bound transferrin (TfR) and low-density lipoprotein (LDL), after releasing their iron or LDL, recycle back to the plasma membrane from early endosomes or recycling endosomes (Scott et al. 2014). Proteins destined for endocytic degradation at the lysosomes are multimono- or poly-ubiquitinated primarily at Lys63 residue and thus recognized by the ESCRT complex. With the help of the ESCRT complex players, the ubiquitinated cargo proteins are selectively sorted by budding and subsequently pinched off from the endosomal membrane into the luminal space, leading to the formation of multivesicular bodies (MVBs) (Raiborg and Stenmark 2009). MVBs are late endosomes having a number of internal vesicles. Fully matured $\mathrm{MVB} /$ late endosomes then fuse with lysosomes to form endosome-lysosome hybrids where their contents are degraded by lysosomal enzymes (Hyttinen et al. 2013). The trafficking of epidermal growth factor receptor (EGFR) is a classic example for this endocytic degradative pathway.

The ESCRT proteins are multisubunit protein complexes that sort endocytosed ubiquitinated proteins such as misfolded plasma membrane proteins, activated growth factors, hormone and cytokine receptors in MVBs. The ESCRT machinery consists of four complexes, ESCRT-0, -I, II and -III along with several accessory components (Hanson et al. 2009).

Ubiquitin dependant cargo sorting was first identified in vacuolar protein sorting (VPS) mutants of Saccharomyces cerevisiae (Katzmann et al. 2002). ESCRT proteins are conserved across species and their depletion cause impaired formation of intralumenal vesicles (ILV), abnormal genesis of MVBs as well as inhibition of lysosomal degradation of protein. ESCRT-0 is the first complex to interact with ubiquitinated cargoes and is associated with endosomal membrane. It is composed of HRS (Hepatocyte growth factor receptor substrate) and STAM (signal-transducing adaptor molecule) subunits. HRS binds to endosomal lipid phosphatidylinositol 3-phosphate $(\operatorname{PtdIns}(3) \mathrm{P})$ and thereby recruited to endosomal membranes (Raiborg et al. 2001). ESCRT-0 binds to multiple ubiquitin molecules (through ubiquitininteracting motifs) and also has clathrin binding domains at C-terminus that helps to increase their local concentration in clathrin coats at the endosomal membrane (Raiborg and Stenmark 2009; Raiborg et al. 2001). Depletion of HRS affects accumulation of internal vesicles within MVBs and inhibits degradation of EGFR (Razi and Futter 2006). Further, ubiquitinated proteins accumulate at early endosomes (Filimonenko et al. 2007).

The next player in this sequential flow of events is the ESCRT-I complex, that consists of 
four subunits - TSG101, Tumor suppressor gene 101 (VPS23 in yeast), VPS28, VPS37 and MVB12 (Morita et al. 2007; Chu et al. 2006). This complex contains a headpiece (that binds to ESCRT-II), a rigid 13-nm stalk and an endpiece that contains the ubiquitin-binding and ESCRT0-binding ubiquitin E2 variant (UEV) domain. The UEV domains of TSG101 and VPS23 bind to PSAP-like (Proline-Serine/Threonine-Alanine- Proline) motifs in HRS. Along with additional interactions, these motifs contribute to the endosomal recruitment of ESCRT-I (Raiborg and Stenmark 2009). TSG101 is required for the generation of EGF-stimulated MVBs by aiding formation of stable vacuolar domains with early endosomes. Depletion of TSG101 perturbs sorting of EGFR to the internal vesicles of the MVBs as well as cargo recycling to the cell surface or the Golgi complex (Doyotte et al. 2005). Absence of TSG101 also prevents formation of the HRS/TSG101 complex and therefore affects recruitment of EGFR in MVBs. This further perturbs the membrane reorganization required for formation of the endosome vacuole and its attendant tubules. This ultimately results in the presence of enlarged vacuoles folded into multicisternal structures resembling the 'Class E' compartments found in VPS mutants of Saccharomyces cerevisiae (Doyotte et al. 2005). The inactivation of TSG101 leads to tubulation of the vacuolar domains of early endosomes and perturbation in MVB formation - thus potentially inhibiting degradation of EGF (Razi and Futter 2006).

Further, depletion of TSG101 affects multiple post-endosomal sorting pathways that include transport of cathepsin D to lysosomes via golgi apparatus as well as transport of transferrin receptor to cell surface. The latter indicates that Class E early endosomes are defective in retrieval of membrane from the trans-golgi network (TGN). Recycling of the transferrin receptor from vacuolar regions (that give rise to the multicisternal endosomes) is affected whilst recycling from tubulo-vesicular regions remains unperturbed. In the absence of TSG101, the clathrin binding domain of HRS remains unexposed, therefore the EGFRcontaining multicisternal structures lack clathrin-coated domains. HRS may bind to ubiquitinated cargo but is unable to sequester it efficiently in the absence of the scaffold that clathrin would otherwise provide and therefore the transport to and from the golgi apparatus is perturbed (Doyotte et al. 2005).

This is followed by the ESCRT-II complex proteins, composed of one EAP45 (VPS36 in yeast), one EAP30 (VPS22 in yeast) and two EAP20 (VPS25 in yeast) subunits (Im and Hurley 2008). The $\mathrm{N}$ terminus GLUE domain (GRAM-like ubiquitin-binding) in EAP45 binds to 
ubiquitin (Alam et al. 2006), as well as 3-phosphorylated phosphoinositides endosomal membrane. ESCRT-II complex also binds to the ESCRT-I VPS28 carboxy-terminal domain subunit through a helix immediately C-terminal to the GLUE domain. Finally, the ESCRT-III complex is constituted of CHMP6 (VPS20 in yeast), CHMP4A, B, C (VPS32/Snf7 in yeast) CHMP3 (VPS24 in yeast) and CHMP2A/B (VPS2 in yeast). VPS20 interacts with VPS25 subunit of ESCRT-II and thus gets recruited to the endosomal membrane (Teo et al. 2004). VPS20 then interacts with VPS32 and mediates the assembly of other ESCRT-III subunits (Teis et al. 2008). Finally, VPS2 associates with the VPS24 cap to mediate recruitment of the ATPase VPS4 (Teis et al. 2008; Saksena et al. 2009). VPS4 catalyzes the dissociation of all three ESCRT complexes from the endosome (Obita et al. 2007; Babst et al. 1998) and thus plays a critical role in the cargo sorting/recycling pathway. The enzyme DOA4 deubiquitinates cargoes prior to sorting into MVB vesicles (Amerik et al. 2000). Mutations in CHMP2B are associated with frontotemporal dimentia (FTD) and amyotrophic lateral sclerosis (ALS). It is reported that missense mutation in CHMP2B(T104N) delays degradation of EGFR. CHMP2B(T104N) tends to accumulate in early and late endosomes; this probably acts as a dominant negative mutant because of its tight association with the other ESCRT-III subunit, mSnf7-2 (mammalian Snf7). Moreover it shows less association with the VPS4 ATPase, resulting in reduced dissociation from ESCRT complexes, a crucial step of MVB sorting (Han et al. 2012).

\subsection{Autophagic degradation and ESCRTs}

The other arm of lysosomal degradation comprises the autophagosomal pathway, that in turn is of three types - i) macroautophagy, ii) microautophagy and iii) chaperone mediated autophagy (García-Arencibia et al. 2010). This review will focus only on the macroautophagic pathway since our understanding of the role of ESCRTs and its accessory proteins is still premature for the other two types of autophagy. For this reason, autophagy is synonymously used for macroautophagy throughout rest of the review. By this process, aged organelles, insoluble misfolded protein complexes that form aggregates or aggresomes and relatively long lived and functional proteins are sequestered in a double membrane (isolation membrane/phagophore) primarily originating from mitochondria-associated endoplasmic reticulum (ER) membrane (Lamb et al. 2013; Hamasaki et al. 2013). There are evidence of other cellular components like golgi bodies, the plasma membrane, and recycling endosomes that contribute to the formation of 
autophagosomes (Lamb et al. 2013). Upon closure of the phagophore, double membranous autophagosomes are formed.

Autophagy requires participation of several ATG proteins (autophagy related gene), including LC3 protein (microtubule-associated protein 1 light-chain 3; ATG 8 in yeast) in its lipidated form referred to as LC3-II. ATG8 family contains two subfamilies that contain at least seven proteins in humans. The MAP1LC3 or LC3 group includes MAP1LC3A, MAP1LC3B, and MAP1LC3C, and the $\gamma$-aminobutyric acid type A receptor-associated protein (GABARAP) group includes GABARAP, GABARAP-like1 (GABARAPL1), GABARAPL2 and GABARAPL3 (Kalvari et al. 2014; Wang et al. 2015). Another protein, p62/SQSTM1, a polyubiquitin-binding protein interacts with LC3-II and both get degraded at the lysosomes (Pankiv et al. 2007). Apart from p62, TOLLIP (Toll interacting protein), NDP52 (Nuclear Dot Protein $52 \mathrm{kDa}$ ), OPTN (Optineurin) and NBR1 (neighbor of BRCA1 gene 1) also bind to ubiquitinated cargoes and LC3-II (Behrends and Fulda. 2012; Lu et al. 2014). Both LC3 and p62 are considered as markers of autophagic flux. Along with ubiquitin dependant protein degradation, there are reports suggesting that degradation of proteins by macroautophagic pathway may occur in a ubiquitin independant manner, like the interaction of BiP with p62 (ChaMolstad et al. 2015). Similarly $\beta$-catenin and Huntingtin interact directly with LC3B and GABARAPL1, respectively, to trigger degradation by macroautophagy (Petherick et al. 2013; Ochaba et al. 2014). Proteins such as AP2 and c-Cbl, both lacking ubiquitin binding domain can draw substrates into macroautophagy owing to their ability to bind to ATG8 family members (Tian et al. 2013; Sandilands et al. 2011).

Autophagosomes then either fuse directly with lysosomes or can fuse with MVB to form amphisomes. The amphisomes then fuse with lysososmes to form autolysosomes where finally the degradation of cargo takes place (Fader and Colombo. 2009). Autophagy acts as survival pathway that helps to maintain cellular health by recycling cellular components during nutrient starvation and also by degrading protein aggregates which are otherwise detrimental for cells. It is also involved in restriction of pathogen invasion, regulation of cell death and plays an important role in diseases like cancer and neurodegeneration.

Depletion of various subunits of the various ESCRT complexes leads to an increase in the number of autophagosomes generated/accumulated in a cell at a given time. This increase in abundance can either be due to an increase in the synthesis of autophagosomes or due to blocked 
fusion of autophagosomes with lysosomes. The blocked autophagosome - lysosome fusion further leads to inhibition of autolysosome formation as is quite evident in ESCRT depleted cells (Rusten et al. 2007; Filimonenko et al. 2007; Lee et al. 2007).

Loss of the ESCRT-0 subunit HRS impairs maturation of autophagosomes. In HRS depleted HeLa cells, reduced occurance of colocalisation between GFP-LC3-positive GAS (surrounding group A Streptococcus) structures and lysosomal marker, Lysosomal-associated membrane protein 1 (LAMP1) is reported (Tamai et al. 2007). In transgenic mice neurons, deletion of Hrs gene leads to accumulation of ubiquinated proteins, such as glutamate receptors and autophagyregulating protein, p62 in hippocampal CA3 neurons and cerebral cortex with advancing age, providing an evidence for the role of an ESCRT protein in modulating autophagy in vivo (Tamai et al. 2008).

Similarly, while depletion of the ESCRT-I protein TSG101, leads to accumulation of ubiquitinated proteins on early endosomes, it also parallelly results in an increase in p62 and LC3 positive autophagosomes and reduction in the formation of autolysosomes. This indicates a blockage in autophagic degradation (Filimonenko et al. 2007). A recent report from our laboratory shows that multi-monoubiquitinated TSG101 is essential for the fusion of (i) amphisomes with lysosomes and (ii) MVBs with lysosomes (Majumder and Chakrabarti 2015). Accumulation of autophagosomes due to blocked maturation of the ESCRT-I proteins is also evidenced in the fly system. VPS28 null mutation in Drosophila melanogaster shows accumulation of autophagosome and decreased formation of autolysosome (Rusten et al. 2007). ATG8 aggregates (demarcating the autophagosomes) accumulate in distinct structured other than LAMP1 positive late endosomes and lysosomes.

In cells depleted of the ESCRT-II component VPS22, ubiquitinated proteins accumulate near lysosomes in non-endosomal compartments indicating blocked autophagic degradation (Filimonenko et al. 2007). Similary, in Drosophila, knocking down of the ESCRT-II protein VPS25, causes accumulation of autophagosomes (Rusten et al. 2007; Lee et al. 2007).

There are a number of evidences suggesting the involvement of ESCRT-III proteins in the fusion of autophagosome with lysosome in cell lines and fly models. Functional depletion of the ESCRT-III components, mSnf7-2 or CHMP2B causes extensive accumulation of autophagosomes and multilamellar bodies that leads to neuronal cell loss (Lee et al. 2007), suggesting that its dysfunction interferes with fusion of autophagosomes with vesicles of the 
endocytic pathway. Phenotypically this resembles depletion of ESCRT-I components in cell culture systems (Doyotte et al. 2005).

Further, it has been recently reported that another ESCRT-III component CHMP4B helps autophagosomal degradation of extranuclear chromatin during cytokinesis. CHMP4B is seen to colocalize with both chromosome bridges and micronuclei. During cytokinesis, autophagosomes and lysosomes accumulate around CHMP4B-positive micronuclei. Cataract associated mutation in CHMP4B abolishes this phenotype and results in impaired autophagic degradation (Sagona et al. 2014). In Drosophila, inactivation of ESCRT-III by overexpression of mutant CHMP2B also results in accumulation of autophagosomes. In flies, null mutations in the CHMP4B homolog, VPS32 causes accumulation of autophagosomes again implying blocked fusion between autophagosomes and endolysosomes (Rusten et al. 2007).

\section{ESCRT regulatory proteins}

So far it has been established that the ESCRT complex proteins have an important role in endolysosomal fusions, autophagosomal maturation and amphisomal-lysosomal fusions. These complexes are in turn modulated by the ESCRT regulatory proteins to enable their efficient activity. These accessory proteins regulate the ESCRT subunits by multiple mechanisms, failing which functioning of the latter is compromised and often reflected as blocked fusion events in either of the lysosomal degradative pathways (Table 1).

\section{1. Fab1}

The endosomal PtdIns(3)P 5-kinase, Fab1 participates in maturation of autolysosomes. Lack of Fab1 does not perturb formation of amphisomes, it results in the accumulation of these vesicles owing to blockage in later fusion events as seen in Drosophila melanogaster (Rusten et al. 2007). It has been shown that while depletion of ESCRT complex proteins results in accumulation of ubiquitinated cargo inside cells, signifying blocked endocytic trafficking, Fab1 depletion results in accretion of non-ubiquitinated cargo. This has led to the speculation that Fab1 does not affect the initial steps of trafficking; it rather participates in the later events culminating at the autolysosomes. It was initially hypothesized to be due to inefficient acidification of the endosomal compartments (Rusten et al. 2006; Nicot et al. 2006). However, very recently it has been reported that Fab1 may not be involved in governing the steady-state 
pH of lysosomes or yeast vacuoles (similarly acidic in $\mathrm{pH}$ ) (Ho et al. 2015). It may play a role in stabilizing lysosomal $\mathrm{pH}$ during cellular stress. Lacking further validation, it is prudent to state that Fab1 does play a role in regulating the fusion of late endosomes with lysosomes.

\subsection{VPS4}

The AAA ATPase VPS4 (vacuolar protein sorting) interacts with ESCRT-III components and is necessary for ESCRT-III function. It is required to disassemble ESCRT-III from membranes and recycle its individual subunits back into the cytoplasm (Adell and Teis. 2011). ATPase deficient VPS4 acts as dominant negative mutant in endosomal sorting and transport (Babst et al. 1998). Recent evidence directly links VPS4 to ILV biogenesis, whereby coordinated binding of VPS4 to ESCRT-III subunits, Snf7 and VPS2 directly couples ESCRT-III disassembly to membrane neck constriction during MVB biogenesis (Adell et al. 2014). Incidentally this is an example where an ESCRT regulatory protein is involved in vesicular fission from the cell membrane. Further, in Drosophila, loss of VPS4 function causes the accumulation of autophagosomes due to inhibited fusion with the endolysosomal system. The severity of phenotype caused by dominant negative mutant of VPS4 is milder than that caused by null mutations in the ESCRT-I and II proteins, VPS28 and VPS25. This indicates a lower penetrance of VPS4 dominant negative phenotypes than ESCRT mutants (Rusten et al. 2007). Similarly, expression of the dominant negative mammalian homolog of VPS4, SKD1DN (referred to as SKD1E235Q mutant) in cell lines perturbs endo-lysosomal and endosome-autophagosomal transport. As a secondary effect, generation of autophagosomes is elevated possibly due to a feedback control loop (Nara et al. 2002).

\subsection{ALIX}

ALIX [formerly apoptosis-linked gene 2- interacting protein X/PDCD6IP (programmed cell death 6 interacting protein)] is a mammalian ESCRT-adaptor cytosolic protein, having a yeast homologue Brol (resistance to osmotic shock protein-1) (Bissig and Gruenberg 2014). ALIX interacts with the ESCRT-I and ESCRT-III proteins TSG101 and CHMP4 (Snf7 in yeast), respectively (Bissig and Gruenberg 2014; Falguie'res et al. 2008). It participates in recruiting of cargoes to the early endosomes by virtue of its interaction with TSG101 and also aids in the formation of ILVs in late endosomes through its association with CHMP4. ALIX specifically 
interacts with lysobisphosphatidic acid (LBPA) on late endosomes though a flexible loop in its N-terminal Bro1 domain and thus potentiates formation of ILVs in late endosomes (Bissig et al. 2013; Matsuo et al. 2004). However there are reports to suggest that ALIX is dispensable for the MVB targeting of the canonical cargo EGFR and its depletion does not affect degradation of EGFR (Cabezas et al. 2005). Depletion of ALIX leads to accumulation of MVBs in the perinuclear region. ALIX via its $\mathrm{V}$ domains interacts with the actin-binding protein cortactin and therefore depletion of ALIX leads to changes in the actin cytoskeletal organization (Cabezas et al. 2005, Murrow et al. 2015). Knockdown of ALIX affects basal macroautophagy in a nutrient rich condition; accumulation of autophagosomes due to their blocked maturation into autolysosomes is also observed. The interaction of ALIX with ATG12-ATG3 plays a key role in this pathway (Murrow et al. 2015).

ALIX and Brol may bind to ubiquitinated cargo proteins as well as participate in sorting of non-ubiquitinated proteins such as Protease-activated receptor 1(PAR1) (Dores et al. 2012) and CD4 (Amorim et al. 2014). PAR1, a G protein-coupled receptor (GPCR) for thrombin is an example where internalization of the protein or its sorting into ILVs of MBVs occurs without binding to ESCRT-0 and -I components (HRS and TSG101). Rather it binds to ALIX by virtue of which it is targeted to MVBs; finally culminating in lysosomal degradation (Dores et al. 2012). Mammalian arrestin-domain containing protein-3 (ARRDC3) regulates ALIX function in GPCR sorting through ubiquitination - activation of PAR1 leads to ubiquitination of ALIX in presence of ARRDC3. This ubiquination requires WWP2, E3 ubiquitin ligase that interacts with ARRDC3. Depletion of either of ARRDC3 or WWP2 inhibits ALIX ubiquitination and blocks the interaction between ALIX-activated PAR1 and CHMP4B. It hence affects lysosomal degradation of PAR1 (Dores et al. 2015). Similarly NEF (Negative Regulatory Factor) induced ubiquitin-independent targeting of CD4, a type I transmembrane glycoprotein expressed on the surface of helper T-lymphocytes and cells of the macrophage/monocyte lineage is also modulated by ALIX. Targeting of CD4 to MVB requires both ALIX and TSG101 (Amorim et al. 2014). Further, ALIX along with the ESCRT-III subunits CHMP4 and CHMP2 are essential for some of the retroviral budding (Schmidt and Teis 2012). The successive recruitment of ALIX, ESCRT-III and VPS4 is required to severe the neck of the maturing virus - again providing an example where an ESCRT protein is involved in fission of viral buds from the cell surface. 


\subsection{MGRN1}

Mahogunin Ring Finger1 (MGRN1), a ubiquitin E3 ligase whose loss of function leads to lateonset neurodegeneration is also implicated in lysosomal dysfunction (Kim et al. 2007; He et al. 2003; Chakrabarti and Hegde 2009). This ligase interacts with and multi-momoubiquitinates the ESCRT-I protein, TSG101 (Kim et al. 2007). In cell culture systems, functional depletion of MGRN1 compromises its post-translational modification of TSG101, perturbs endolysosomal degradation, increases the number of autophagosomes and affects autophagosomal-lysosomal degradation (Majumder and Chakrabarti 2015; Kim et al. 2007). A simultaneous blockage is thus detected in both the arms of lysosomal degradation. Multi-monoubiquitinated TSG101 is shown to be responsible for enabling fusion events between (i) MVBs and lysosomes, (ii) amphisomes and lysosomes. Hence, depletion of MGRN1 affects both types of lysosomal degradation. Interestingly blocked monoubiquitination of TSG101 in MGRN1 depleted condition does not alter either sorting of cargoes into MVBs or generation of amphisomes by fusion between MVBs and autophagosomes. This recent report suggests a role for ESCRT-I proteins in late fusion events with lysosomes without affecting initial events (Majumder and Chakrabarti 2015). This also indicates that modification of ESCRT proteins plays one of key role for regulating their functionality.

\subsection{TMEM106B}

TMEM106B is a late endosomal-lysosomal type-II glycoprotein implicated in frontotemporal lobar degeneration (FLTD) and ALS (Brady et al. 2013; Jun et al. 2015). Its overexpression results in enlargement of lysosomes and impairs endo-lysosomal degradation. Cellular studies reveal that endogenous TMEM106B associates with both the wild type and mutant forms of ESCRT-III, CHMP2B on early/late endosomes. Single nucleotide polymorphism (SNP) variant T185-TMEM106B associates with CHMP2B and reduces autophagic flux. The dynamic and transient interaction of the ESCRT complex including CHMP2B with TMEM106B affects autophagosomal degradation; this impaired autophagic flux could in turn potentiate neurotoxicity. Further, TMEM106B expression also modulates the translocation of transcription factor EB, which controls autophagy and lysosomal biogenesis under stress. This could in turn be another possible link between TMEM106B and autophagic fusion (Stagi et al. 2014). 


\subsection{Atrogin-1}

Atrogin-1, a muscle-specific ubiquitin ligase is associated with degradation of signaling proteins involved in cardiac hypertrophy (Bodine et al 2001; Gomes et al 2001; Zaglia et al. 2014). Atrogin-1 knockout mice develop age related cardiomyopathy. These mice also fail to degrade the ESCRT- III protein CHMP2B resulting in autophagic impairment, intracellular protein aggregate accumulation, unfolded protein response activation, and subsequent cardiomyocyte apoptosis, all of which increase progressively with age. Accumulation of CHMP2B due to loss of Atrogin-1 compromises endosome-lysome maturation and fusion of autophagosome and lysosomes. The degradation of CHMP2B by its regulatory protein Atrogin-1 could mediate a crosstalk between the ubiquitin-proteasome and the autophagosome-lysosome systems (Zaglia et al. 2014).

\subsection{ATG12-ATG3}

ATG3 has recently been identified as a target for ATG12, which is a core component of the autophagy pathway (Murrow et al. 2015). ATG12-ATG3 complex interacts with the ESCRT associated protein ALIX (also known as PDCD6IP). In cell culture systems, deletion of ATG12ATG3 results in compromised basal autophagic flux, accumulation of perinuclear late endosomes and impaired endolysosomal trafficking. Further, ATG12-ATG3 controls various ALIX-dependent processes including late endosome distribution, exosome biogenesis, and viral budding. Under nutrient rich condition, deletion of ATG3-ATG12 complex leads to accumulation of autophagosomes due to their blocked maturation into autolysosomes. These findings shed light on a mechanism which ESCRTs and the associated protein ALIX could mediate regulation of fusion between autophagosome and lysosome, while also regulating the endo-lysosomal degradation pathway (Murrow et al. 2015). Conversely, ALIX deficiency specifically impairs basal autophagy similarly to that of ATG12-ATG3. Overall, these results identify an interconnection between the core autophagy and ESCRT machineries that facilitates basal autophagic flux and multiple ALIX-associated activities at the late endosome.

\subsection{Syntaxin 13}

Syntaxin 13 (STX13) is a member of the Q-SNARE (Soluble NSF Attachment Protein Receptor having glutamine $(\mathrm{Q})$ as the central amino acid at core binding domain motif) family of proteins. 
It is recently reported that Drosophila STX13 exhibits a strong genetic interaction with mutant CHMP2B, the ESCRT-III protein. Depletion of STX13 function results in the enrichment of multilamellar structure and blockage in autophagic pathways in mammalian cell culture systems. Accumulation of STX13 is reported when CHMP2B is mutated ( $\mathrm{Lu}$ et al. 2013). Knockdown of the binding partner of STX13, Vtila also leads to accumulation of LC3-positive punta and impaired autophagic flux. These results suggest that STX13 is a genetic modifier of ESCRT-III dysfunction and participates in the maturation of phagophores into closed autophagosomes (Lu et al. 2013).

\subsection{Protein kinase CK2 $\alpha$}

Protein kinase CK2 $\alpha$ has diverse functions, amongst which it also regulates the function of ESCRT-III proteins in MVB sorting. It is involved in the phosphorylation of the ESCRT-III subunits CHMP3 and CHMP2B, as well as of VPS4B/SKD1 (ATPase) at acidic phosphosites (serine residue) present in them. Down regulation of $\mathrm{CK} 2 \alpha$ does not affect localization of ESCRT-III subunits on endosomes but impairs degradation of EGF (Salvi et al. 2014). ESCRTIII and VPS4 have been shown to play distinct role in vesicular fusion. Their inactivation leads to blockage in both autophagic and endocytic degradation pathways owing to perturbation in fusion events. Thus CK2a may be speculated to affect the phosphorylation status of CHMPs and VPS4B proteins and this in turn could alter vesicular fusion events in an indirect manner. This would add another level of complexity to the ESCRT associated endosomal trafficking.

\section{Discussion:}

In this review, we have discussed in details the role of ESCRTs and their regulatory proteins in primarily lysosomal fusion events. The impaired function of ESCRT subunits and their regulatory proteins affect trafficking of cargo, formation of MVB, maturation of autophagosomes/endosomes and their fusion with lyosomes (Figure 1). However, recent evidences also suggest that ESCRT proteins can perturb autophagic pathway without affecting formation of MVBs. Inactivation of ESCRT proteins result in accumulation of autophagosomes because of blocked fusion between autophagosome and lysosome (Figure 2). The mechanism of fusion processes between lysosomes and autophagosomes/amphisomes is poorly understood in 
neurodegenerative diseases. However, this may represent a critical property in these diseases since the fusion step determines the efficiency of the autophagy flux. The same applies for endocytic pathway where after uptake and sorting of the cargo, degradation depends on fusion competence of MVBs with lysosomes.

Interestingly, the significance of ESCRT protein function in regulating autophagy is reported across species - mammals (human and mouse), Drosophila, Saccharomyces (Rusten et al. 2007; Lee et al. 2007; Rusten and Stenmark 2009; Manil-Segalén et al. 2012; Mizushima 2015; Liu and Du 2015). Studies in these systems have elegantly demonstrated that the impairment of endocytic and autophagic pathways may lead to disturbances in cellular homeostasis. A larger number of diseases like Alzheimer's disease, Niemann Pick Disease, Huntington's disease and ALS are termed as lysosomal diseases and pathogenesis of all these diseases are closely linked to lysosomal dysfunction -- a significant proportion of these are due to perturbations of fusion events in the autophagosomal and endocytic pathways. Therefore it is worth exploring how these pathways are regulated in late stages of the fusion cascades.

Acknowledgement: This work was supported by the "Integrative Biology on Omics Platform Project", intramural funding of the Department of Atomic Energy (DAE), Government of India.

Conflict of interest: The authors declare no conflict of interest, financial or otherwise. 


\section{References:}

Adell, M.A., and Teis, D. 2011. Assembly and disassembly of the ESCRT-III membrane scission complex. FEBS Lett. 585 (20): 3191-3196.

Adell, M.A., Vogel, G.F., Pakdel, M., Müller, M., Lindner, H., Hess. M.W., et al. 2014. Coordinated binding of Vps4 to ESCRT-III drives membrane neck constriction during MVB vesicle formation. J Cell Biol. 205 (1): 33-49.

Agromayor, M., Martin-Serrano, J. 2013. Knowing when to cut and run: mechanisms that control cytokinetic abscission. Trends Cell Biol. 23(9): 433-441.

Alam, S.L., Langelier, C., Whitby, F.G., Koirala, S., Robinson, H., Hill, C.P., and Sundquist, W.I. 2006. Structural basis for ubiquitin recognition by the human ESCRT-II EAP45 GLUE domain. Nature Struct. Mol. Biol. 13 (11): 1029-1030.

Amerik, A.Y., Nowak, J., Swaminathan, S., and Hochstrasser, M. 2000. The Doa4 deubiquitinating enzyme is functionally linked to the vacuolar protein-sorting and endocytic pathways. Mol. Biol. Cell. 11 (10): 3365-3380.

Amorim, N.A., da Silva, E.M., de Castro, R.O., da Silva-Januário, M.E., Mendonça, L.M., Bonifacino, J.S., et al. 2014. Interaction of HIV-1 Nef Protein with the Host Protein Alix Promotes Lysosomal Targeting of CD4 Receptor. J Biol Chem. 289 (40): 27744-27756.

Babst, M., Katzmann, D.J., Snyder, W.B., Wendland, B., and Emr, S.D. 2002. Endosomeassociated complex, ESCRT-II, recruits transport machinery for protein sorting at the multivesicular body. Dev Cell. 3 (2): 283-239.

Babst, M., Wendland, B., Estepa, E.J., Emr, S.D. 1998. The Vps4p AAA ATPase regulates membrane association of a $\mathrm{Vps}$ protein complex required for normal endosome function.

EMBO J. 17 (11): 2982-2993.

Bache, K.G., Brech, A., Mehlum, A., and Stenmark, H. 2003. Hrs regulates multivesicular body formation via ESCRT recruitment to endosomes. J Cell Biol. 162 (3): 435-442.

Bartusch, C., Prange, R. 2016. ESCRT Requirements for Murine Leukemia Virus Release. Viruses. 8(4). pii: E103.

Behrends, C., Fulda, S. 2012. Receptor proteins in selective autophagy. Int J Cell Biol. 2012:673290. 
Bissig, C., and Gruenberg, J. 2014. ALIX and the multivesicular endosome: ALIX in Wonderland. Trends Cell Biol. 24 (1): 19-25.

Bissig, C., Lenoir, M., Velluz, M.C., Kufareva, I., Abagyan, R., Overduin, M., et al. 2013.Viral infection controlled by a calcium-dependent lipid-binding module in ALIX. Dev. Cell 25 (4): 364-373.

Bodine, S.C., Latres, E., Baumhueter, S., Lai, V.K., Nunez, L., Clarke, B.A., et al. 2001. Identification of ubiquitin ligases required for skeletal muscle atrophy. Science. 294 (5547): 1704-1708.

Brady, O.A., Zheng, Y., Murphy, K., Huang, M., and Hu, F. 2013.The frontotemporal lobar degeneration risk factor, TMEM106B, regulates lysosomal morphology and function, Hum Mol Genet 22 (4): 685-695.

Caballe, A., Wenzel, D.M., Agromayor, M., Alam, S.L., Skalicky, J.J., Kloc, M., et al. 2015 ULK3 regulates cytokinetic abscission by phosphorylating ESCRT-III proteins. Elife. 4:e66547.

Cabezas, A., Bache, K.G., Brech, A., and Stenmark, H. 2005. Alix regulates cortical actin and the spatial distribution of endosomes. J. Cell Sci. 118 (12): 2625-2635.

Carlton, J.G., Martin-Serrano, J. 2007. Parallels between cytokinesis and retroviral budding: A role for the ESCRT machinery. Science. 316(5833): 1908-1912.

Chakrabarti, O., and Hegde, R.S. 2009. Functional Depletion of Mahogunin by Cytosolically Exposed Prion Protein Contributes to Neurodegeneration. Cell. 137 (6): 1136-1147.

Cha-Molstad, H., Sung, K.S., Hwang, J., Kim, K.A., Yu, J.E., Yoo, Y.D., et al. 2015. Aminoterminal arginylation targets endoplasmic reticulum chaperone BiP for autophagy through p62 binding. Nat Cell Biol. 17(7):917-929.

Chu, T., Sun, J., Saksena. S., and Emr, S.D. 2006. New component of ESCRT-I regulates endosomal sorting complex assembly, J. Cell Biol. 175 (5): 815-823.

Dores, M.R., Chen, B., Lin, H., Soh, U.J., Paing, M.M., Montagne, W.A., et al. 2012. ALIX binds a YPX3L motif of the GPCR PAR1 and mediates ubiquitin-independent ESCRT-III/MVB sorting. J. Cell Biol. 197 (3): 407-419. 
Dores, M.R., Lin, H., J Grimsey, N., Mendez, F., and Trejo, J. 2015. The $\alpha$-arrestin ARRDC3 mediates ALIX ubiquitination and G protein-coupled receptor lysosomal sorting. Mol Biol Cell. 26 (25): 4660-4673.

Doyotte, A., Russell, M.R., Hopkins, C.R., Woodman, P.G. 2005. Depletion of TSG101 forms a mammalian "Class E" compartment: a multicisternal early endosome with multiple sorting defects. J Cell Sci. 118 (14):3003-3017.

Fader, C.M., and Colombo, M.I. 2009. Autophagy and multivesicular bodies: two closely related partners. Cell Death Differ. 16 (1): 70-78.

Falguières, T., Luyet, P.P., Bissig, C., Scott, C.C., Velluz, M.C., and Gruenberg, J. 2008. In vitro budding of intralumenal vesicles into late endosomes is regulated by Alix and Tsg101. Mol. Biol. Cell. 19 (11): 4942-4955.

Filimonenko, M., Stuffers, S., Raiborg, C., Yamamoto, A., Malerod, L., Fisher, E.M., et al. 2007. Functional multivesicular bodies are required for autophagic clearance of protein aggregates associated with neurodegenerative disease. J. Cell Biol. 179 (3): 485-500.

Ganley, I.G. 2013. Autophagosome maturation and lysosomal fusion. Essays Biochem. 55: 6578.

García-Arencibia, M., Hochfeld, W.E., Toh, P.P., and Rubinsztein, D.C. 2010. Autophagy, a guardian against neurodegeneration. Seminars in Cell \& Developmental Biology. 21 (7): 691698.

Gomes, M.D., Lecker, S.H., Jagoe, R.T., Navon, A., and Goldberg, A.L. 2001. Atrogin-1, a muscle-specific F-box protein highly expressed during muscle atrophy. Proc Natl Acad Sci U S A. 98 (25): 14440-14445.

Gruenberg, J., and Stenmark, H. 2004. The biogenesis of multivesicular endosomes. Nat Rev Mol Cell Biol. 5 (4): 317-323.

Hamasaki, M., Furuta, N., Matsuda, A., Nezu, A., Yamamoto, A., Fujita, N. 2013. Autophagosomes form at ER-mitochondria contact sites. Nature. 495 (7441): 389-393.

Han, J.H., Ryu, H.H., Jun, M.H., Jang, D.J., and Lee, J.A. 2012. The functional analysis of the CHMP2B missense mutation associated with neurodegenerative diseases in the endo-lysosomal pathway. Biochem Biophys Res Commun. 421 (3): 544-549. 
Hanson, P.I., Shim, S. and Merrill, S.A. 2009. Cell biology of the ESCRT machinery. Curr. Opin. Cell Biol. 21 (4): 568-574.

He, L., Lu, X.Y., Jolly, A.F., Eldridge, A.G., Watson, S.J., Jackson, P.K. et al. 2003. Spongiform degeneration in mahoganoid mutant mice. Science. 299 (5607):710-712.

Henne, W.M., Stenmark, H., Emr, S.D. 2013. Molecular mechanisms of the membrane sculpting ESCRT pathway. Cold Spring Harb Perspect Biol. 5(9). pii: a016766.

Ho, C.Y., Choy, C.H.1, Wattson, C.A., Johnson, D.E., and Botelho R.J. 2015. The Fab1/PIKfyve phosphoinositide phosphate kinase is not necessary to maintain the $\mathrm{pH}$ of lysosomes and of the yeast vacuole, J Biol Chem. 290 (15): 9919-9928.

Huotari, J., and Helenius, A. 2011. Endosome maturation. EMBO J. 30 (17): 3481-500.

Hurley, J.H. 2010. The ESCRT Complexes, Crit Rev Biochem Mol Biol. 45 (6): 463-487.

Hurley, J.H. 2015. ESCRTs are everywhere. EMBO J. 34(19): 2398-2407.

Hyttinen, J.M., Niittykoski, M., Salminen, A., and Kaarniranta, K., 2013. Maturation of autophagosomes and endosomes: a key role for Rab7. Biochim Biophys Acta. 1833(3): 503-510.

Im, Y.J., and Hurley, J.H. 2008. Integrated structural model and membrane targeting mechanism of the human ESCRT-II complex. Dev. Cell 14(6): 902-913.

Jun, M.H., Han, J.H., Lee, Y.K., Jang, D.J., Kaang, B.K., and Lee, J.A. 2015. TMEM106B, a frontotemporal lobar dementia (FTLD) modifier, associates with FTD-3-linked CHMP2B, a complex of ESCRT-III. Molecular Brain. 8:85.

Katzmann, D.J., Odorizzi, G., and Emr, S.D. 2002. Receptor downregulation and multivesicularbody sorting. Nature Rev. Mol. Cell Biol. 3(12): 893-905.

Kalvari, I., Tsompanis, S., Mulakkal, N.C., Osgood, R., Johansen, T., Nezis, I.P. et al. 2014. iLIR: A web resource for prediction of Atg8-family interacting proteins. Autophagy. 10(5):913925.

Kim, B.Y., Olzmann, J.A., Barsh, G.S., Chin, L.S., Li, L. 2007. Spongiform neurodegenerationassociated E3 ligase Mahogunin ubiquitylates TSG101 and regulates endosomal trafficking. Mol Biol Cell. 18 (4): 1129-1142.

Lamb, C.A., Yoshimori, T., and Tooze, S.A. 2013. The autophagosome: origins unknown, biogenesis complex. Nature Reviews Molecular Cell Biology. 14 (12): 759-774. 
Lee, J.A., Beigneux, A., Ahmad, S.T., Young, S.G., Gao, F.B., 2007. ESCRT-III dysfunction causes autophagosome accumulation and neurodegeneration. Curr. Biol. 17 (18): 1561-1567.

Liu, X.M., and Du, L.L. 2015. A selective autophagy pathway takes an unconventional route. Autophagy11(12):2381-2382.

Lu, K., Psakhye, I., Jentsch, S. 2014. Autophagic clearance of polyQ proteins mediated by ubiquitin-Atg8 adaptors of the conserved CUET protein family. Cell. 158(3):549-563.

Lu, Y., Zhang, Z., Sun, D., Sweeney, S.T., Gao, F.B., 2013. Syntaxin 13, a genetic modifier of mutant CHMP2B in frontotemporal dementia, is required for autophagosome maturation. Mol Cell. 52(2):264-271.

Luzio, J.P., Gray, S.R., Bright. N.A., 2010. Endosome-lysosome fusion. Biochem Soc Trans. 38(6):1413-1416.

Majumder, P., and Chakrabarti, O. 2015. Mahogunin regulates fusion between amphisomes/MVBs and lysosomes via ubiquitination of TSG101. Cell Death Dis. 6: e1970.

Manil-Segalén, M., Lefebvre, C., Culetto, E., and Legouis, R. 2012. Need an ESCRT for autophagosomal maturation? Commun Integr Biol. 5 (6): 566-571.

Matsuo, H., Chevallier, J., Mayran, N., Le Blanc, I., Ferguson, C., Fauré, J. et al. 2004. Role of LBPA and Alix in multivesicular liposome formation and endosome organization. Science. 303 (5657): 531-534.

Mayor, S., and Pagano, R.E. 2007. Pathways of clathrin-independent endocytosis. Nat Rev Mol Cell Biol. 8 (8): 603-612.

Metcalf, D., and Isaacs, A.M. 2010. The role of ESCRT proteins in fusion events involving lysosomes, endosomes and autophagosomes. Biochem Soc Trans. 38 (6): 1469-1473.

Mizushima, N. 2015. Nbr1, a Receptor for ESCRT-Dependent Endosomal Microautophagy in Fission Yeast. Mol Cell. 59(6): 887-889.

Morita, E., Sandrin, V., Alam, S.L., Eckert, D.M., Gygi, S.P., and Sundquist, W.I. 2007. Identification of human MVB12 proteins as ESCRT-I subunits that function in HIV budding, Cell Host Microbe. 2 (1): 41-53.

Moreau, K., Renna, M., Rubinsztein, D.C., 2013. Connections between SNAREs and autophagy. Trends Biochem Sci. 38 (2): 57-63. 
Murrow, L., Malhotra, R., and Debnath, J. 2015. ATG12-ATG3 Interacts with Alix to Promote Basal Autophagic Flux and Late Endosome Function. Nat Cell Biol. 17 (3): 300-310.

Nara, A., Mizushima, N., Yamamoto, A., Kabeya, Y., Ohsumi, Y., Yoshimori, T. 2002. SKD1 AAA ATPase-dependent endosomal transport is involved in autolysosome formation. Cell Struct. Funct. 27(1): 29-37.

Nicot, A.S., Fares, H., Payrastre, B., Chisholm, A.D., Labouesse, M., and Laporte. J. 2006. The phosphoinositide kinase PIKfyve/ Fab1p regulates terminal lysosome maturation in Caenorhabditis elegans. Mol. Biol. Cell. 17 (7): 3062-3074.

Obita, T., Saksena, S., Ghazi-Tabatabai, S., Gill, D.J., Perisic, O., Emr, S.D., and Williams, R.L. 2007. Structural basis for selective recognition of ESCRT-III by the AAA ATPase Vps4. Nature 449 (7163): 735-739.

Ochaba, J., Lukacsovich, T., Csikos, G., Zheng, S., Margulis, J., Salazar, L., et al. 2014. Potential function for the Huntingtin protein as a scaffold for selective autophagy. Proc Natl Acad Sci U S A. 111(47):16889-16894.

Pankiv, S., Clausen, T.H., Lamark, T., Brech, A., Bruun, J.A., and Outzen, H. 2007. p62/SQSTM1 binds directly to Atg8/LC3 to facilitate degradation of ubiquitinated protein aggregates by autophagy. J Biol Chem. 282 (33): 24131-24145.

Petherick, K.J., Williams, A.C., Lane, J.D., Ordóñez-Morán, P., Huelsken, J., Collard, T.J., et al. 2013 Autolysosomal $\beta$-catenin degradation regulates Wnt-autophagy-p62 crosstalk. EMBO J. 32(13):1903-1916.

Raiborg, C., Bache, K. G., Mehlum, A., Stang, E., and Stenmark, H. 2001. Hrs recruits clathrin to early endosomes. EMBO J. 20 (17): 5008-5021.

Raiborg, C., and Stenmark, H. 2009. The ESCRT machinery in endosomal sorting of ubiquitylated membrane proteins. Nature. 458 (7237): 445-452.

Razi, M., and Futter, C.E. 2006. Distinct roles for Tsg101 and Hrs in multivesicular body formation and inward vesiculation. Mol Biol Cell. 17 (8): 3469-3483.

Rusten, T.E., Rodahl, L.M., Pattni, K., Englund, C., Samakovlis, C., Dove, S., et al., 2006. Fab1 phosphatidylinositol 3-phosphate 5-kinase controls trafficking but not silencing of endocytosed receptors. Mol. Biol. Cell. 17 (9): 3989-4001.

Rusten, T.E., and Simonsen, A. 2008. ESCRT functions in autophagy and associated disease. Cell Cycle. 7 (9):1166-1172. 
Rusten, T.E., and Stenmark, H. 2009. How do ESCRT proteins control autophagy? J Cell Sci. 122 (13): 2179-2183.

Rusten, T.E., Vaccari, T., Lindmo, K., Rodahl, L.M., Nezis, I.P.,Sem-Jacobsen, C., et al. 2007. ESCRTs and Fab1 regulate distinct steps of autophagy. Curr. Biol. 17 (20): 1817-1825.

Sagona, A.P., Nezis, I.P., Stenmark, H. 2014. Association of CHMP4B and autophagy with micronuclei: implications for cataract formation. Biomed Res Int. 2014: 974393.

Saksena, S., Wahlman, J., Teis, D., Johnson, A.E., and Emr, S.D. 2009. Functional reconstitution of ESCRT-III assembly and disassembly. Cell. 136 (1): 97-109.

Salvi, M., Raiborg, C., Hanson, P.I., Campsteijn, C., Stenmark, H., and Pinna, L.A. 2014. CK2 involvement in ESCRT-III complex phosphorylation. Archives of Biochemistry and Biophysics, 545: 83-91.

Sandilands, E., Serrels, B., McEwan, D.G., Morton, J.P., Macagno, J.P., McLeod, K., et al. 2011. Autophagic targeting of Src promotes cancer cell survival following reduced FAK signalling. Nat Cell Biol. 14(1):51-60.

Schmidt, O., and Teis, D. 2012. The ESCRT machinery. Curr Biol. 22 (4): R116-120.

Scott, C.C., Vacca, F., and Gruenberg, J. 2014. Endosome maturation, transport and functions, Seminars in Cell \& Developmental Biology. 31: 2-10.

Spang, A. 2009. On the fate of early endosomes, Biol. Chem. 390 (8): 753-759.

Stagi, M., Klein, Z.A., Gould, T.J., Bewersdorf, J., and Strittmatter, S.M. 2014. Lysosome size, motility and stress response regulated by fronto-temporal dementia modifier TMEM106B. Mol Cell Neurosci. 61: 226-240.

Tamai, K., Tanaka, N., Nara, A., Yamamoto, A., Nakagawa, I., Yoshimori, T., et al. 2007. Role of Hrs in maturation of autophagosomes in mammalian cells. Biochem. Biophys. Res. Commun. 360 (4): 721-727.

Tamai, K., Toyoshima, M., Tanaka, N., Yamamoto, N., Owada, Y., Kiyonari, H., et al. 2008. Loss of hrs in the central nervous system causes accumulation of ubiquitinated proteins and neurodegeneration. Am J Pathol. 173 (6): 1806-1817. 
Teis, D., Saksena, S., and Emr, S.D. 2008. Ordered assembly of the ESCRT-III complex on endosomes is required to sequester cargo during MVB formation. Dev. Cell. 15 (4): 578-589.

Teo, H., Perisic, O., González, B., and Williams, R.L. 2004. ESCRT-II, an endosome-associated complex required for protein sorting: crystal structure and interactions with ESCRT-III and membranes. Dev. Cell 7 (4): 559-569.

Tian, Y., Chang, J.C., Fan, E.Y., Flajolet, M., Greengard, P. 2013. Adaptor complex AP2/PICALM, through interaction with LC3, targets Alzheimer's APP-CTF for terminal degradation via autophagy. Proc Natl Acad Sci U S A. 110(42):17071-17076.

Votteler, J., Sundquist, W.I. 2013. Virus budding and the ESCRT pathway. Cell Host Microbe. 14(3): 232-241

Wang, D.W., Peng, Z.J., Ren, G.F., Wang, G.X. 2015. The different roles of selective autophagic protein degradation in mammalian cells. Oncotarget. 6 (35): 37098-37116.

Zaglia, T., Milan, G., Ruhs, A., Franzoso, M., Bertaggia, E., Pianca, N., et al. 2014. Atrogin-1 deficiency promotes cardiomyopathy and premature death via impaired autophagy. J Clin Invest. 124 (6): 2410-2424. 
Table 1: Various ESCRT regulatory proteins modulates role of ESCRTs in autophagy and endocytic degradation.

\begin{tabular}{|c|c|c|c|}
\hline Regulatory Proteins & $\begin{array}{c}\text { Effect on substrate } \\
\text { ESCRTs }\end{array}$ & $\begin{array}{c}\text { Substrates/ } \\
\text { downstream targets }\end{array}$ & Pathways affected \\
\hline $\begin{array}{l}\text { Fab1, endosomal } \\
\text { PtdIns(3)P 5-kinase }\end{array}$ & Unknown & Unknown & $\begin{array}{l}\text { i) Late endosome- } \\
\text { lysosome fusion } \\
\text { ii) Amphisome- } \\
\text { lysosome fusion }\end{array}$ \\
\hline AAA ATPase VPS4 & $\begin{array}{l}\text { Disassemble ESCRT- } \\
\text { III from membranes }\end{array}$ & Snf7 and VPS2 & $\begin{array}{l}\text { i) Formation of ILV in } \\
\text { MVB } \\
\text { ii)Autophagosome- } \\
\text { endolysosome fusion }\end{array}$ \\
\hline $\begin{array}{l}\text { ALIX (apoptosis- } \\
\text { linked gene 2- } \\
\text { interacting protein X) }\end{array}$ & $\begin{array}{c}\text { Interacts with } \\
\text { ESCRT-I and } \\
\text { ESCRT-III }\end{array}$ & TSG101 and CHMP4 & i) MVB biogenesis \\
\hline $\begin{array}{l}\text { Mahogunin RING } \\
\text { Finger 1(MGRN1) }\end{array}$ & $\begin{array}{c}\text { Multi- } \\
\text { monoubiquitination }\end{array}$ & TSG101 & $\begin{array}{l}\text { i) MVB-Lysosome } \\
\text { fusion } \\
\text { ii) Amphisosome- } \\
\text { lysosome fusion }\end{array}$ \\
\hline TMEM106B & $\begin{array}{l}\text { Tight association of } \\
\text { mutant form with } \\
\text { ESCRT-III leads to } \\
\text { impaired function }\end{array}$ & СНMP2B & $\begin{array}{c}\text { i) Endosome- } \\
\text { lysosome degradation } \\
\text { ii) Autophagosome- } \\
\text { lysosome fusion }\end{array}$ \\
\hline Atrogin 1 & $\begin{array}{l}\text { Proteosomal } \\
\text { degradation of } \\
\text { ESCRT-III }\end{array}$ & CHMP2B & $\begin{array}{l}\text { i) Endosome- } \\
\text { lysosome degradation } \\
\text { ii) Autophagosome- } \\
\text { lysosome fusion }\end{array}$ \\
\hline ATG12-ATG3 & Interaction with ALIX & ALIX & $\begin{array}{l}\text { i) Endolysosomal } \\
\text { trafficking } \\
\text { ii) Autophagosome- } \\
\text { lysosome fusion at } \\
\text { basal condition }\end{array}$ \\
\hline Syntaxin 13 & $\begin{array}{l}\text { Genetic interaction } \\
\text { with mutant CHMP2B }\end{array}$ & CHMP2B & $\begin{array}{l}\text { i) Maturation of } \\
\text { phagophores into } \\
\text { autophagosomes } \\
\text { ii) Accumulation of } \\
\text { multimellar structure }\end{array}$ \\
\hline Protein kinase CK2 $\alpha$ & Phosphorylation & $\begin{array}{c}\text { CHMP3, CHMP2B } \\
\text { VPS4B/SKD1 }\end{array}$ & $\begin{array}{l}\text { i) Endosomal } \\
\text { degradation }\end{array}$ \\
\hline
\end{tabular}




\section{Box 1: Fusion events where ESCRTs participate}

ESCRTs and their regulatory proteins affect lyososomal degradation via multiple routes:

$>$ Perturbed formation of ILVs affect MVB biogenesis.

$>$ Blocked maturation of phagophore / phagophore closure to generate autophagososme.

Formation of amphisomes.

- Fusion of amphisosomes / autophagosomes / late endosomes with lysosomes. 


\section{FIGURE LEGENDS}

Figure 1. ESCRTs and regulatory proteins participate along the endocytic and autophagic pathways to regulate various vesicular fusion events.

Cytosolic cargoes are sequestered in phagophore which matures in autophagosomes. Membrane bound cargoes are internalized into early endosomes. ESCRT complexes $(\nabla)$ are recruited to the early endosomes and aid formation of MVBs. They also participate in phagophore closure. Endocytic and autophagic vesicles either individually fuse with lysosomes or fuse with each other to form amphisomes, aided by various ESCRTs and their accessory proteins. Amphisomes then fuse with lysosomes again utilizing these proteins in the various fusion events, marked by arrows.

ESCRT proteins are labeled in black. ESCRT regulatory proteins are marked in red. LC3-II is marked by $\nabla$.

Abbreviations: ESCRT, Endosomal sorting complex required for transport; MVB, Multivesicular body; VPS, Vacuolar protein sorting; STX, Syntaxin.

Reference list for various proteins cited in figure 1:

1) Tamai et al. 2007; 2) Lu et al. 2013; 3) Razi and Futter 2006; 4) Doyotte et al. 2005; Razi and Futter 2006; 5) Han et al. 2012; 6) Bissig et al. 2013; Matsuo et al. 2004; 7) Nara et al. 2002; Rusten et al. 2007; 8) Majumder and Chakrabarti 2015; 9) Rusten et al. 2007.

\section{Figure 2. Depletion of ESCRT and accessory proteins lead to blocked autophagic degradation.}

Depletion of ESCRT subunits and/or their regulatory proteins cause accumulation of autophagososmes due to compromised downstream fusion events.

Dotted arrows denote compromised presence or total absence of events.

Reference list for various proteins cited in figure 2 :

1) Filimonenko et al. 2007; 2) Rusten et al. 2007; 3) Rusten et al. 2007; Lee et al. 2007; 4) Lee et al. 2007; Rusten et al. 2007; 5) Sagona et al. 2014; Rusten et al. 2007; 6) Nara et al. 2002; 7) Murrow et al. 2015; 8) Majumder and Chakrabarti 2015; 9) Zaglia et al. 2014 


\section{Figure 1}

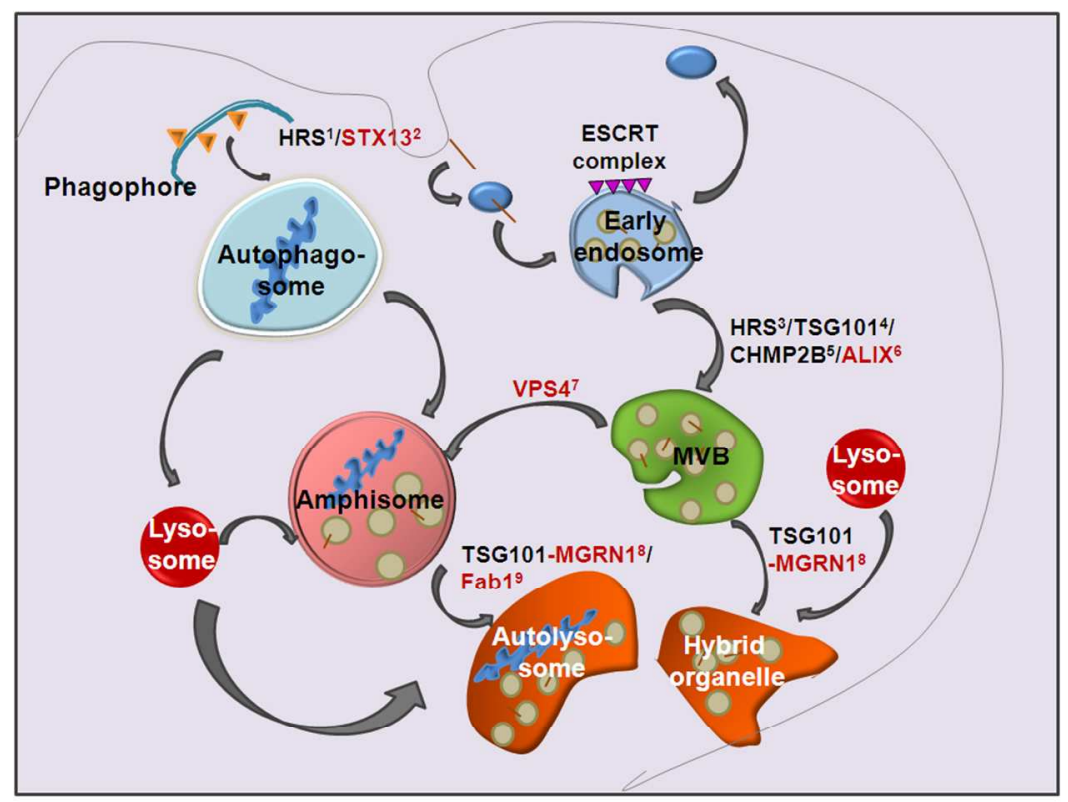

ESCRTs and regulatory proteins participate along the endocytic and autophagic pathways to regulate various vesicular fusion events.

$101 \times 76 \mathrm{~mm}(300 \times 300 \mathrm{DPI})$ 


\section{Figure 2}

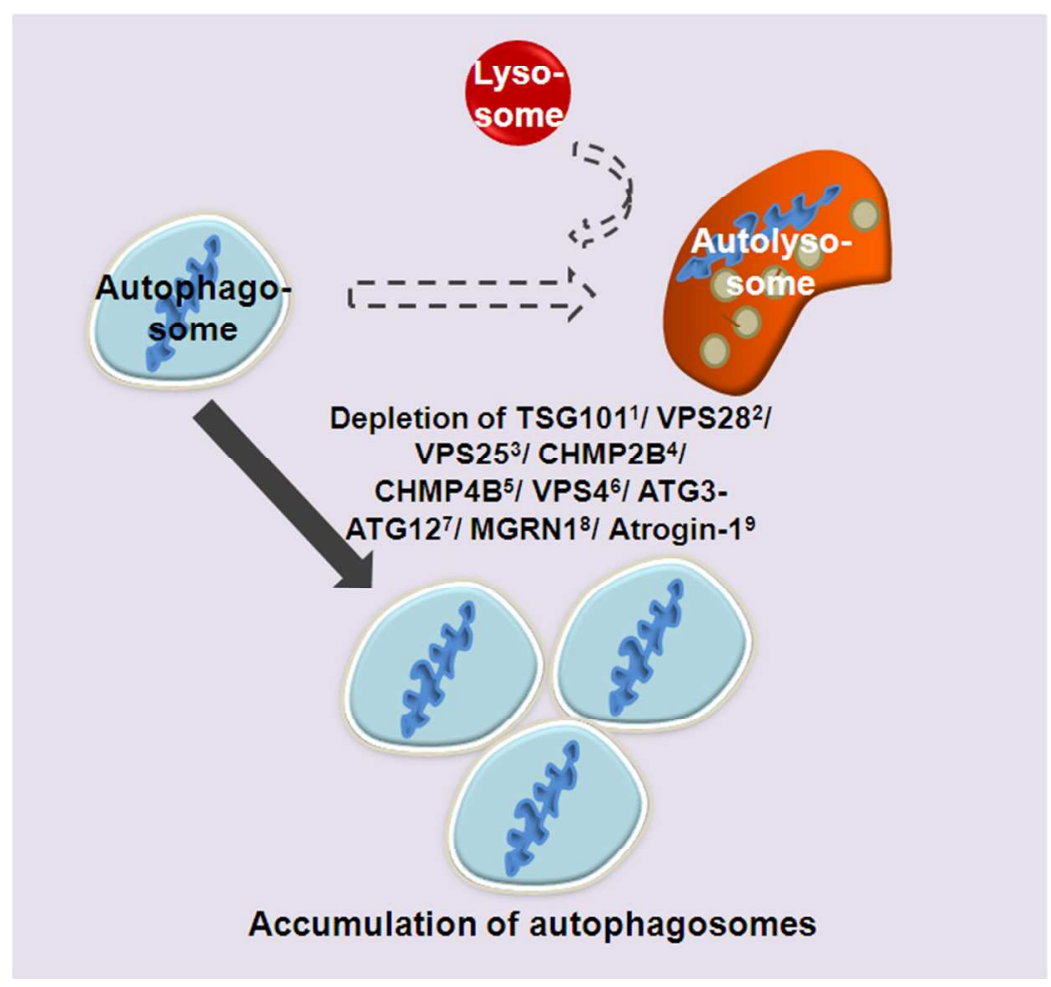

Depletion of ESCRT and accessory proteins lead to blocked autophagic degradation.

$76 \times 76 \mathrm{~mm}(300 \times 300$ DPI $)$ 